Case Report

\title{
Refractory Abdominal Pain in a Patient with Chronic Lymphocytic Leukemia: Be Wary of Acquired Angioedema due to C1 Esterase Inhibitor Deficiency
}

\author{
Abdullateef Abdulkareem (iD, ${ }^{1}$ Ryan S. D'Souza, ${ }^{2}$ Joshua Mundorff, ${ }^{1}$ Pragya Shrestha, \\ Oluwaseun Shogbesan $\left(\mathbb{1},{ }^{1}\right.$ and Anthony Donato $\mathbb{1}^{1}$ \\ ${ }^{1}$ Department of Medicine, Reading Hospital, West Reading, PA, USA \\ ${ }^{2}$ Department of Anesthesiology, Mayo Clinic Hospital, Rochester, MN, USA \\ Correspondence should be addressed to Abdullateef Abdulkareem; dayo.abdulkareem@readinghealth.org
}

Received 26 September 2017; Revised 1 November 2017; Accepted 19 November 2017; Published 10 January 2018

Academic Editor: Kate Khair

Copyright ( $\odot 2018$ Abdullateef Abdulkareem et al. This is an open access article distributed under the Creative Commons Attribution License, which permits unrestricted use, distribution, and reproduction in any medium, provided the original work is properly cited.

\begin{abstract}
Acquired angioedema due to $\mathrm{C} 1$ inhibitor deficiency (C1INH-AAE) is a rare and potentially fatal syndrome of bradykinin-mediated angioedema characterized by episodes of angioedema without urticaria. It typically manifests with nonpitting edema of the skin and edema in the gastrointestinal (GI) tract mucosa or upper airway. Edema of the upper airway and tongue may lead to life-threatening asphyxiation. C1INH-AAE is typically under-diagnosed because of its rarity and its propensity to mimic more common abdominal conditions and allergic reactions. In this article, we present the case of a 62-year-old male with a history of recently diagnosed chronic lymphocytic leukemia (CLL) who presented to our hospital with recurrent abdominal pain, initially suspected to have Clostridium difficile colitis and diverticulitis. He received a final diagnosis of acquired angioedema due to $\mathrm{C} 1$ esterase inhibitor deficiency due to concomitant symptoms of lip swelling, cutaneous nonpitting edema of his lower extremities, and complement level deficiencies. He received acute treatment with $\mathrm{C} 1$ esterase replacement and icatibant and was maintained on $\mathrm{C} 1$ esterase infusions. He also underwent chemotherapy for his underlying CLL and did not experience further recurrence of his angioedema.
\end{abstract}

\section{Introduction}

Acquired angioedema due to $\mathrm{C} 1$ esterase inhibitor deficiency (C1INH-AAE) is a rare and potentially fatal disorder caused by acquired consumption of $\mathrm{C} 1$ esterase inhibitor. Studies estimate a prevalence rate between 1 in 100,000 and 1 in 500,000 patients, although it may be higher as the condition is commonly unrecognized [1]. It may manifest with nonpitting edema of the skin, including the skin of the face, lips, limbs, or genitals, abdominal pain secondary to edema of the gastrointestinal mucosa, and severe life-threatening edema of the upper airway and oral mucosa [2].

\section{Case Presentation}

A 62-year-old male with a two-month history of recurrent hospital admissions for abdominal pain presented to the emergency room with colicky, generalized abdominal pain. He also reported new asymmetric swelling of his upper and lower lips. He denied associated difficulty swallowing or breathing. He had no previous history of angioedema, food allergies, or new medications. He denied any fever, chills, malaise, trauma, nausea, vomiting, diarrhea, constipation, hematochezia or melena, weight loss, sick contacts, neurological deficits, or recent travel history. Of note, he had never taken angiotensin-converting enzyme inhibitors (ACEi) or angiotensin receptor blocker medications. He was a former smoker and quit 14 years ago and reported only occasional alcohol intake. He had no family history of angioedema. This was the third hospital admission for abdominal pain episodes within six weeks, with prior episodes spontaneously resolving within a few days.

Six weeks prior to current presentation, a computed tomography (CT) scan of the abdomen for a prior bout of 
this illness revealed diffuse small bowel thickening with mild adjacent mesenteric fat stranding and a thickened, nodular terminal ileum and cecum. He also had a positive Clostridium difficile stool toxin test. He was treated with metronidazole for $C$. difficile colitis although diarrhea was not a prominent symptom. Two weeks prior to current presentation, he was admitted for recurrent abdominal pain for which another CT was performed, and on this one, he was found to have severe jejunal edema, new proctocolitis, and several enlarged mesenteric and inguinal lymph nodes. The lymph nodes were biopsied and found to have CD5-positive cells consistent with a new diagnosis of chronic lymphocytic leukemia/small lymphocytic lymphoma (CLL/SLL), later confirmed by bone marrow biopsy. The patient was again treated for presumed $C$. difficile colitis, due to another positive stool toxin test, this time with oral vancomycin. He also received a course of amoxicillin-clavulanate as CT abdomen showed extensive diverticulosis and evidence of sigmoid colitis, introducing the possibility of diverticulitis. Evaluation during that admission included esophagogastroduodenoscopy (EGD) and push enteroscopy. Both were unremarkable except for previously known Barrett's esophagus. Interestingly, he also had asymmetric left lower limb cutaneous swelling and pain during that admission that had an unrevealing workup including a negative Doppler ultrasound.

During the current presentation, vital signs were normal with oxygen saturation at $95 \%$ on room air. Physical examination revealed lungs that were clear to auscultation without stridor. Symmetric upper and lower lip swelling without hives was noted (Figure 1). The central abdomen was mildly tender to palpation two inches above the umbilicus with no guarding or rebound. No edema was noted in the lower extremities during this current admission. All other systems examined were normal.

\section{Investigations}

Complete blood count revealed a white blood count of 4900/ $\mu \mathrm{L}$ (normal: 4,800-10,800/ $\mathrm{L}$ ), hemoglobin of 12.4 (normal: $14-17.5 \mathrm{~g} / \mathrm{dL}$ ), and platelets of 149,000 (normal: 130,000-400,000/ $\mu \mathrm{L}$ ). Lactic acid, sedimentation rate, C-reactive protein, lipase, and liver function tests were normal. Considering the bouts of unexplained bowel edema and swelling lips without hives, a diagnostic workup for angioedema was pursued. Functional C1 esterase inhibitor level was found to be $4 \%$ (normal: $>40 \%$ ), along with $\mathrm{C} 1$ esterase inhibitor antigen of $<3 \mathrm{mg} / \mathrm{dl}$ (normal: $21-39 \mathrm{mg} / \mathrm{dl}$ ), C4 complement $<1.7 \mathrm{mg} / \mathrm{dl}$ (normal: $12-38 \mathrm{mg} / \mathrm{dl}$ ), and low normal C1q level of $113 \mu \mathrm{g} / \mathrm{ml}$ (normal: 109-242 $\mu \mathrm{g} / \mathrm{ml}$ ). C3 complement level was normal at $88.6 \mathrm{mg} / \mathrm{dl}$ (normal: $59-152 \mathrm{mg} / \mathrm{dl}$ ). C. difficile stool toxin test during this admission was negative. Stool samples were also negative for Salmonella, Shigella, Escherichia coli, Campylobacter, Yersinia enterocolitica, Giardia, Vibrio, and Aeromonas. CT abdomen and pelvis (Figure 2) showed jejunal enteritis with progressive duodenitis and stable abdominopelvic lymphadenopathy. A repeat $\mathrm{C} 1 \mathrm{q}$ level performed 6 months later was low at $<50 \mu \mathrm{g} / \mathrm{ml}$ $(109-242 \mu \mathrm{g} / \mathrm{ml})$.

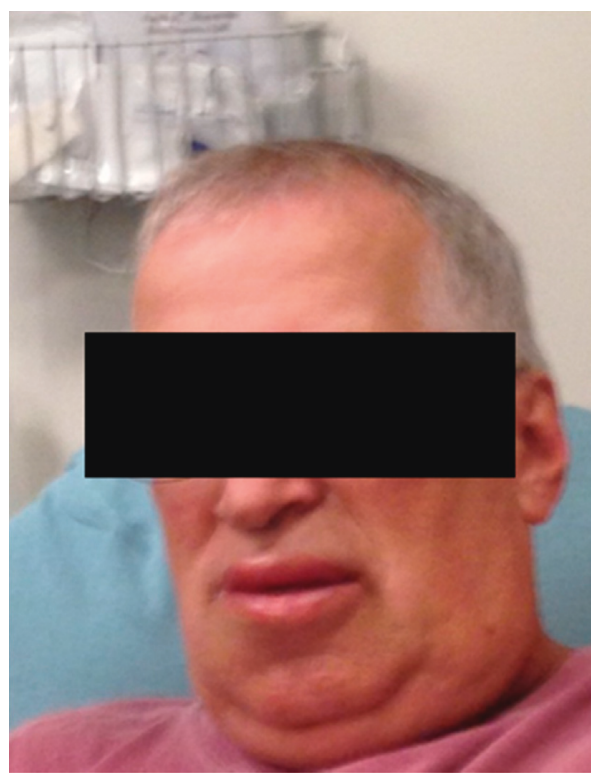

FIgURE 1: Patient with symmetric lip swelling of upper and lower lips.

\section{Differential Diagnosis}

Given the patient's history of positive C. difficile stool tests, a diagnosis of $C$. difficile colitis was initially assigned, although repeat stool toxin assay was negative. Diverticulitis, acute pancreatitis, peptic ulcer disease, acute cholecystitis, and inflammatory bowel syndrome were excluded based on clinical history, negative biochemical and stool tests, EGD, and CT abdomen/pelvis results. Stool studies were negative for bacterial or parasitic infection that could otherwise explain the CT scan findings. Viral gastroenteritis was considered although patient denied any nausea, vomiting, or diarrhea, and symptoms appeared to be recurrent.

Since nonspecific abdominal pain was accompanied with symptoms of lip swelling and recent cutaneous swelling in his lower extremities, a suspicion was formed of angioedema. Allergic angioedema was excluded as the patient denied urticaria and exposure to precipitating agents and had abnormal complement levels in his serum. Similarly, the presence of low C1-INH levels [3] and the absence of ACEi use excluded ACEi-induced angioedema. Hereditary angioedema (HAE) was unlikely because our patient denied a family history of angioedema and experienced onset of symptoms in his 6th decade, as opposed to before the 2nd decade which is more typical of HAE [4].

\section{Treatment}

Patient was urgently administered intravenous methylprednisolone $125 \mathrm{mg}$ and benadryl. Once a diagnosis of acquired $\mathrm{C} 1$ esterase deficiency was made, he received $\mathrm{C} 1$ esterase replacement and icatibant (a bradykinin $\mathrm{B}_{2}$ receptor antagonist). He was subsequently maintained on $\mathrm{C} 1$ esterase replacement therapy and given rescue injections of icatibant for acute exacerbations. He also received outpatient chemotherapy for his CLL/SLL with six cycles of rituximab, cyclophosphamide, 


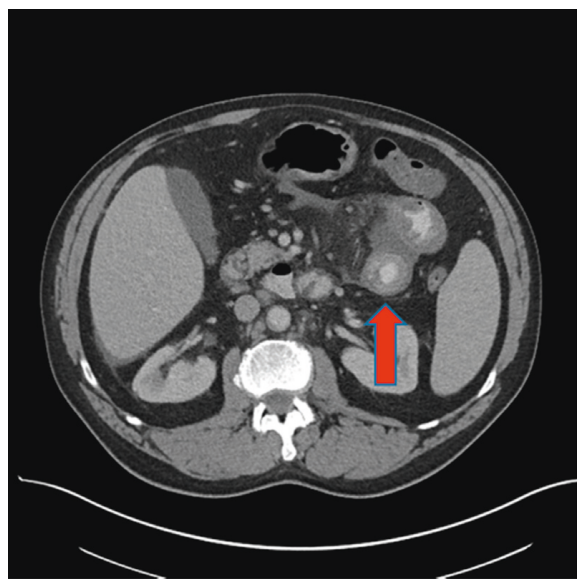

(a)

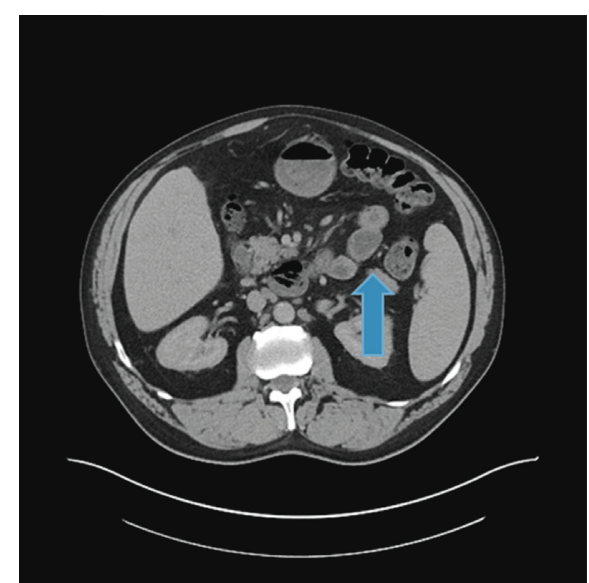

(b)

Figure 2: CT abdomen findings before and after treatment. (a) Before treatment, the patient was noted to have jejunal enteritis, which is indicated by the target sign (red arrow). (b) After treatment, the patient had resolution of jejunitis (blue arrow).

vincristine, and prednisone (R-CVP). He had no further episodes of abdominal pain, lip swelling, or lower extremity swelling. Interval imaging with CT abdomen showed resolution of jejunitis and duodenitis (Figure 2).

\section{Discussion}

Approximately half of patients with C1INH-AAE may have upper airway edema, and consequently death from asphyxiation or anoxic brain injury can occur in up to $30 \%$ of patients $[3,5,6]$. However, more benign and nonspecific presentations of GI angioedema and cutaneous edema may be the only manifesting symptoms. GI symptoms of angioedema include nonspecific colicky abdominal pain, vomiting, diarrhea, and abdominal distension [2], which can be mistaken for common abdominal conditions such as gastroenteritis and diverticulitis. Cutaneous edema may manifest as nonpitting edema of the skin, usually affecting the face but may also involve the lower extremities, mimicking allergic reactions and thrombophlebitis.

Because the diagnosis is extremely uncommon and its symptoms are protean, there is frequently a delay in diagnosis. A retrospective nationwide study in France found a median delay in diagnosis of 10 months [3], whereas a national audit in the United Kingdom showed a diagnostic delay of 5 years [7]. Our patient was diagnosed within only 2 months of symptom onset, but after three hospital admissions and incorrect diagnoses of $C$. difficile colitis and diverticulitis.

The key pathologic abnormality in C1INH-AAE is the acquired deficiency of C1-inhibitor enzyme (C1-INH). A major function of C1-INH is the inhibition of kallikrein, which is a protease that cleaves kininogen and releases bradykinin [8]. Consequently, in C1-INH deficiency, the absence of inhibition of kallikrein leads to increased bradykinin levels, which then facilitate vasodilation and increased tissue permeability, resulting in angioedema flares [8]. C1-INH deficiency in acquired angioedema (AAE) may be caused by excessive consumption due to hyperactivation of the classic complement pathways associated with underlying lymphoproliferative diseases or by autoantibodies (autoimmune mediated). Both lymphoproliferative disorders and autoantibodies frequently coexist in the same patients and one may lead to the other [9-11]. Our patient did not have antibodies tested, but he did have CLL (a lymphoproliferative disease).

Laboratory findings include low C1-INH activity, low C3 and $\mathrm{C} 4$ levels, and autoantibodies to $\mathrm{C} 1-\mathrm{INH}$ in up to $70 \%$ of patients with AAE $[12,13]$. C1q level is typically low in up to $70 \%$ of patients, although it may be normal in early stages of the disease [14]. Our patient had low C1-INH activity, low C1-INH level, low C4 complement, and an initially low-normal level of $\mathrm{C} 1 \mathrm{q}$ but low repeat $\mathrm{C} 1 \mathrm{q}$ levels six months after diagnosis.

Most patients afflicted with C1INH-AAE also have concomitant lymphoproliferative and autoimmune disorders [15]. In one review that included 136 cases of acquired C1 inhibitor deficiency, lymphatic malignancies were identified in $35 \%$, MGUS in 32\%, autoimmune diseases in $8 \%$, with adenocarcinoma, and other malignancies identified in 6\% [15]. Lymphoproliferative disorders and B-cell malignancies are the most commonly associated conditions $[10,16]$, especially splenic marginal zone lymphomas [17]. Associated autoimmune disorders include systemic lupus erythematosus, cryoglobulinemia, and autoimmune hemolytic anemia $[18,19]$. Interestingly, there have also been reports of association with Helicobacter pylori [20]. In our case, the patient had been recently diagnosed with CLL, a non-Hodgkin's lymphoma with known association with C1INH-AAE [13, 21-23].

Primary pharmacologic treatment for acute $\mathrm{C} 1 \mathrm{INH}-$ AAE includes $\mathrm{C} 1$ esterase inhibitor concentrate from human plasma or recombinant $\mathrm{C} 1$ inhibitor concentrate, icatibant (a synthetic bradykinin $\mathrm{B}_{2}$ receptor antagonist), and/or ecallantide (a kallikrein inhibitor). The most widely used therapy is $\mathrm{C} 1$ esterase inhibitor concentrate, although resistance to this treatment over time, necessitating higher doses to control symptoms, has been reported [10].

Importantly, unlike more common causes of angioedema including allergic and drug-induced angioedema, acute presentations of C1INH-AAE typically do not respond 
to corticosteroids, antihistamine, or epinephrine [24]. Prophylactic treatment to prevent recurrent symptoms of C1INH-AAE includes C1 esterase inhibitor concentrate administered every 3-4 days, attenuated androgens (i.e., danazol and stanozolol) and antifibrinolytic agents $[17,25]$. Our index patient received $\mathrm{C} 1$ esterase inhibitor concentrate and icatibant twice weekly prophylaxis with $\mathrm{C} 1$ esterase inhibitor concentrate. He experienced resolution of his symptoms following initiation of that therapy and treatment of his lymphoma with chemotherapy. Other case reports have documented similar improvement in angioedema symptoms following treatment of lymphoma [26].

Physicians caring for patients with unexplained abdominal pain especially with concomitant skin, lip, or airway swelling should consider angioedema as a possibility and should check laboratory testing for confirmation.

\section{Teaching Points}

(1) Wider appreciation of the possibility of C1INH-AAE in refractory recurrent abdominal pain, particularly in patients with lymphoproliferative disorders, could lead to proper and timely medical therapy and avert fatal respiratory complications from laryngeal and upper airway edema.

(2) Abdominal symptoms in C1INH-AAE may mimic gastroenteritis and diverticulitis, whereas nonpitting cutaneous edema may be mistaken for allergic reactions and thrombophlebitis.

(3) Unlike more common causes of angioedema including allergic and drug-induced angioedema, acute presentations of angioedema secondary to $\mathrm{C} 1$ esterase deficiency typically do not respond to corticosteroids, antihistamine, or epinephrine and necessitate treatment with $\mathrm{C} 1$ inhibitor replacement therapy, icatibant, ecallantide, or fresh frozen plasma.

(4) If an underlying associated disease is identified in a patient with CIINH-AAE, including lymphoproliferative malignancy or autoimmune disease, treatment of the underlying disease may lead to symptomatic improvement of C1INH-AAE.

\section{Conflicts of Interest}

The authors declare that there are no conflicts of interest regarding the publication of this paper.

\section{References}

[1] E. Pappalardo, M. Cicardi, C. Duponchel et al., "Frequent de novo mutations and exon deletions in the $\mathrm{C} 1$ inhibitor gene of patients with angioedema," Journal of Allergy and Clinical Immunology, vol. 106, no. 6, pp. 1147-1154, 2000.

[2] M. Cicardi and A. Zanichelli, "Acquired angioedema," Allergy, Asthma and Clinical Immunology, vol. 6, no. 1, p. 14, 2010.

[3] D. Gobert, R. Paule, D. Ponard et al., "A nationwide study of acquired C1-inhibitor deficiency in France: characteristics and treatment responses in 92 patients," Medicine, vol. 95, no. 33, p. e4363, 2016.
[4] M. P. Henao, T. Craig, J. Kraschnewski, and T. Kelbel, "Diagnosis and screening of patients with hereditary angioedema in primary care," Therapeutics and Clinical Risk Management, vol. 12, pp. 701-711, 2016.

[5] G. Dobson, D. Edgar, and J. Trinder, "Angioedema of the tongue due to acquired C1 esterase inhibitor deficiency," Anaesthesia And Intensive Care, vol. 31, no. 1, pp. 99-102, 2003.

[6] K. Bork, K. Siedlecki, S. Bosch, R. E. Schopf, and W. Kreuz, "Asphyxiation by laryngeal edema in patients with hereditary angioedema," Mayo Clinic Proceedings, vol. 75, no. 4, pp. 349-354, 2000.

[7] S. Jolles, P. Williams, and E. Carne, "A UK national audit of hereditary and acquired angioedema," Clinical and Experimental Immunology, vol. 175, no. 1, pp. 59-67, 2014.

[8] T. Caballero, M. L. Baeza, R. Cabañas et al., "Consensus statement on the diagnosis, management, and treatment of angioedema mediated by bradykinin. Part I. Classification, epidemiology, pathophysiology, genetics, clinical symptoms, and diagnosis," Journal of Investigational Allergology and Clinical Immunology, vol. 21, no. 5, pp. 333-347, 2011.

[9] M. Cugno, R. Castelli, and M. Cicardi, "Angioedema due to acquired C1-inhibitor deficiency: a bridging condition between autoimmunity and lymphoproliferation," Autoimmunity Reviews, vol. 8, no. 2, pp. 156-159, 2008.

[10] M. Cicardi, L. C. Zingale, E. Pappalardo, A. Folcioni, and A. Agostoni, "Autoantibodies and lymphoproliferative diseases in acquired C1-inhibitor deficiencies," Medicine, vol. 82, no. 4, pp. 274-281, 2003.

[11] M. A. Wu and R. Castelli, "The Janus faces of acquired angioedema: C1-inhibitor deficiency, lymphoproliferation and autoimmunity," Clinical Chemistry and Laboratory Medicine, vol. 54, no. 2, pp. 207-214, 2016.

[12] M. Cicardi and A. Zanichelli, "Angioedema due to C1 inhibitor deficiency in 2010," Internal and Emergency Medicine, vol. 5, no. 6, pp. 481-486, 2010.

[13] M. Jung and L. Rice, "“Surgical” abdomen in a patient with chronic lymphocytic leukemia: a case of acquired angioedema," Journal of Gastrointestinal Surgery, vol. 15, no. 12, pp. 2262-2266, 2011.

[14] M. Cicardi, "Acquired C1 Inhibitor Deficiency: Clinical Manifestations, Epidemiology, Pathogenesis, and Diagnosis," UptoDate, 2016.

[15] L. C. Zingale, R. Castelli, A. Zanichelli, and M. Cicardi, "Acquired deficiency of the inhibitor of the first complement component: presentation, diagnosis, course, and conventional management," Immunology and Allergy Clinics of North America, vol. 26, no. 4, pp. 669-690, 2006.

[16] M. Cicardi, D. Lambertenghi Deliliers, A. Zanichelli, L. C. Zingale, and R. Castelli, "Lymphoproliferative disease and acquired C1 inhibitor (C1-INH) deficiency," Journal of Allergy and Clinical Immunology, vol. 119, no. 1, p. S279, 2007.

[17] R. Castelli, M. A. Wu, M. Arquati et al., "High prevalence of splenic marginal zone lymphoma among patients with acquired C1 inhibitor deficiency," British Journal of Haematology, vol. 172, no. 6, pp. 902-908, 2016.

[18] P. Cacoub, V. Frémeaux-Bacchi, I. De Lacroix et al., “A new type of acquired $\mathrm{C} 1$ inhibitor deficiency associated with systemic lupus erythematosus," Arthritis and Rheumatism, vol. 44, no. 8, pp. 1836-1840, 2001.

[19] J. Gelfand, G. R. Boss, C. L. Conley, R. Reinhart, and M. M. Frank, "Acquired C1 esterase inhibitor deficiency and angioedema: a review," Medicine, vol. 58, no. 4, pp. 321-328, 1979. 
[20] D. Mukeba, K. Chandrikakumari, J. B. Giot et al., "Autoimmune angioneurotic edema in a patient with Helicobacter pylori infection," Helicobacter, vol. 14, no. 1, pp. 9-11, 2009.

[21] G. Mohyuddin and I. Rabinowitz, "A patient with chronic lymphocytic leukemia and acquired angioedema: correlation of clinical and biochemical response to CLL therapy," Ecancermedicalscience, vol. 7, p. 292, 2013.

[22] N. Klossowski, S. A. Braun, V. von Gruben et al., "Acquired angioedema with $\mathrm{C} 1-\mathrm{INH}$ deficiency and accompanying chronic spontaneous urticaria in a patient with chronic lymphatic B cell leukemia," Der Hautarzt, vol. 66, no. 10, pp. 723-725, 2015.

[23] R. Castelli, A. Zanichelli, M. Cicardi, and M. Cugno, "Acquired C1-inhibitor deficiency and lymphoproliferative disorders: a tight relationship," Critical Reviews in Oncology/Hematology, vol. 87, no. 3, pp. 323-332, 2013.

[24] A. Agostoni, E. Aygören-Pürsün, K. E. Binkley et al., "Hereditary and acquired angioedema: problems and progress: proceedings of the third C1 esterase inhibitor deficiency workshop and beyond," Journal of Allergy and Clinical Immunology, vol. 114, no. 3, pp. S51-S131, 2004.

[25] M. Cicardi, C. Suffritti, F. Perego, and S. Caccia, "Novelties in the diagnosis and treatment of angioedema," Journal of Investigational Allergology and Clinical Immunology, vol. 26, no. 4, pp. 212-221, 2016.

[26] A. Branellec, L. Bouillet, N. Javaud et al., "Acquired C1-inhibitor deficiency: 7 patients treated with rituximab," Journal of Clinical Immunology, vol. 32, no. 5, pp. 936-941, 2012. 


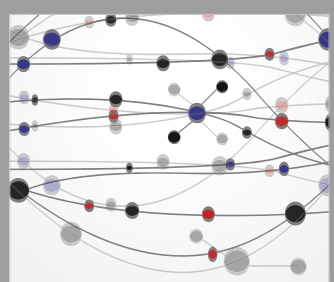

The Scientific World Journal
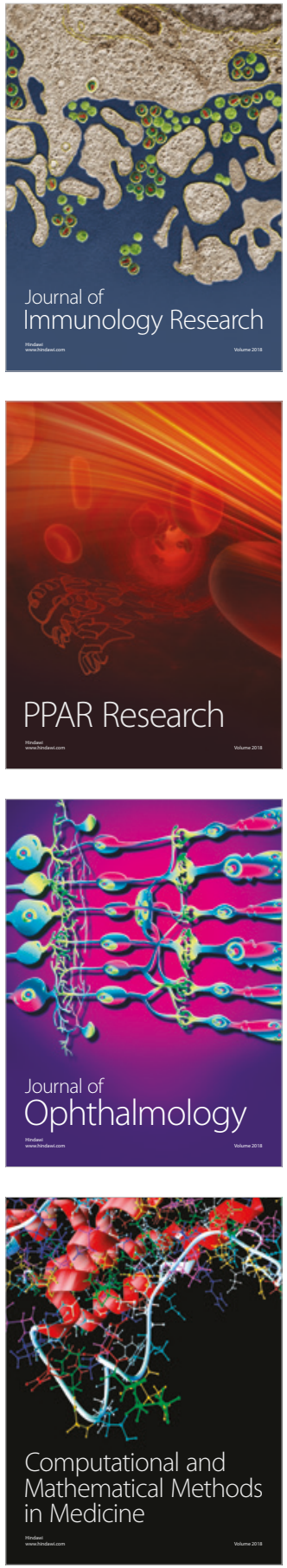

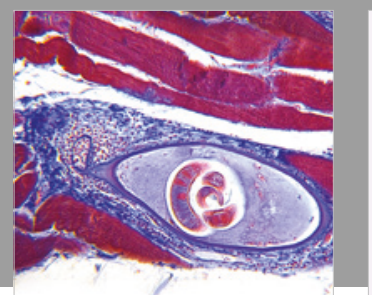

Gastroenterology Research and Practice

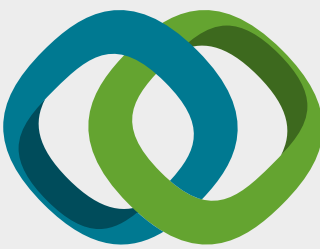

\section{Hindawi}

Submit your manuscripts at

www.hindawi.com
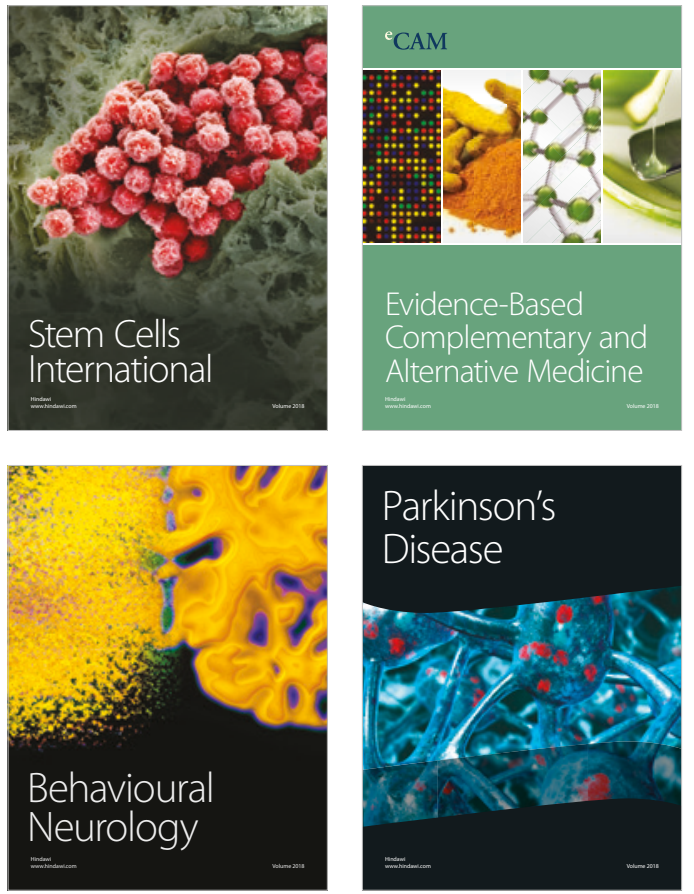

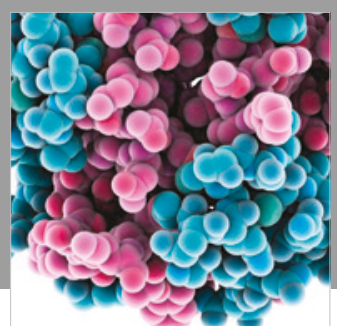

ournal of

Diabetes Research

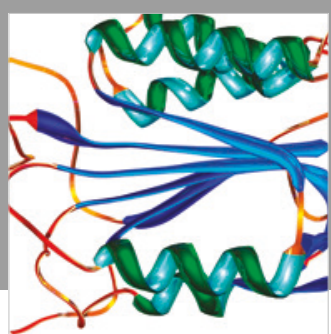

Disease Markers
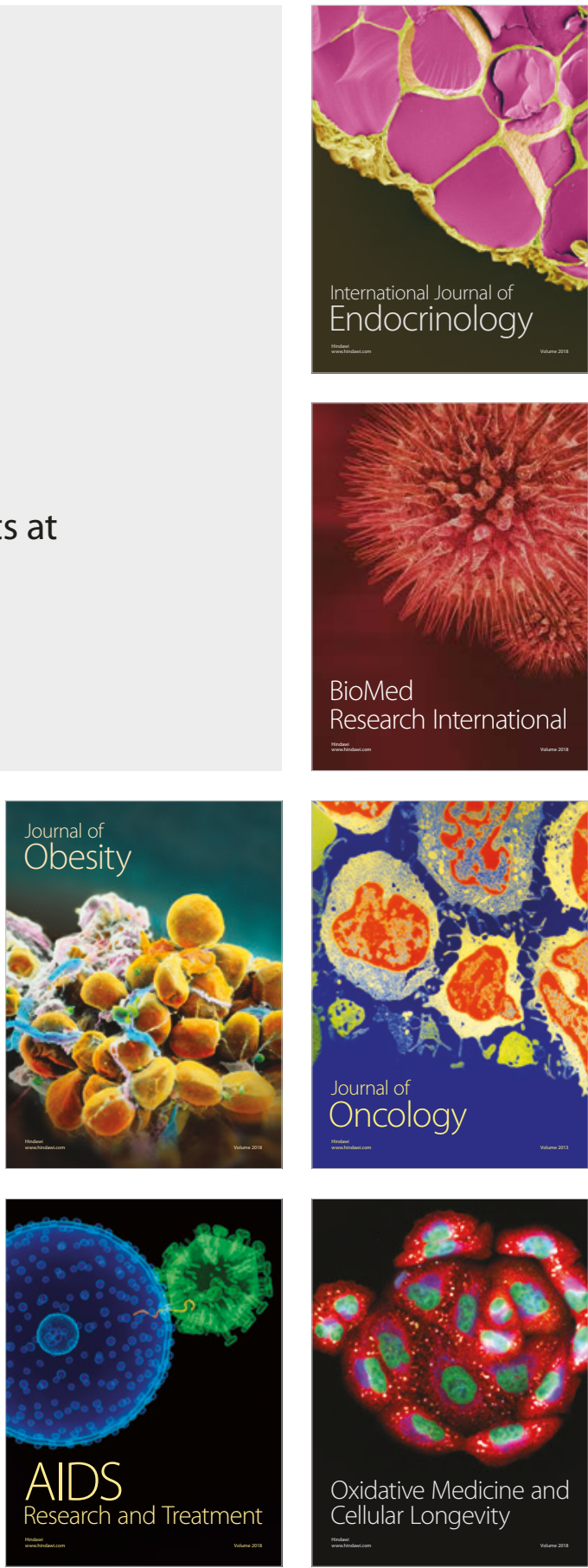